\title{
Ischemic stroke associated with ankylosing spondylitis: an integral part of disease spectrum, or a natural consequence of progressive infirmity?
}

\author{
Sanjay Behari ${ }^{1}$ (D) Suyash Singh ${ }^{1} \cdot$ Kamlesh S. Bhaisora $^{1}$ \\ Received: 11 February 2018 / Accepted: 14 February 2018 / Published online: 1 March 2018 \\ (C) Springer-Verlag GmbH Austria, part of Springer Nature 2018
}

Ankylosing spondylitis (AS), a chronic rheumatic disease with a prevalence ranging from 0.2 to $1.1 \%$ [1, 2], is a manifestation of autoimmunity against the axial skeleton (often leading to spinal immobility) that also affects peripheral joints and extra-articular structures (causing uveitis, inflammatory bowel disease (IBD), psoriasis, and enthesitis). Chronic inflammation, bone destruction, and aberrant bone repair may cause progressive disability. The $3 \mathrm{E}$ Initiative in Rheumatology has stated that AS is likely to be present, if along with chronic back pain of at least 3-month duration, the presence of several of the following features are present: inflammatory back pain, alternating buttock pain, response to non-steroidal anti-inflammatory drugs (NSAIDs), onset of symptoms before the age of 45 years, the presence of peripheral disease manifestations (arthritis, dactylitis, or enthesitis), the presence of confirmed acute anterior uveitis, a positive family history of the disease, the presence of HLA-B27 positivity in the patient, and the diagnosis of sacroiliitis/ spondylitis by imaging [1].

Reports exist regarding the association of AS with an increased risk of ischemic heart disease [3,4], but whether or not this association also translates into a higher incidence of cerebrovascular stroke remains uncertain [5-7]. In a study based in Wales, UK, published in 2012, comparing 1686 AS patients with $1,206,621$ control subjects, there was no increase in the incidence of cerebrovascular disease/stroke rates in patients with AS compared to those without AS, despite there being higher rates of hypertension prevalent in patients with AS [8]. On the other hand, in a study from Sweden, patients with AS $(n=5358)$, rheumatoid arthritis $(n=37,245)$, and those with matched general population subjects $(n=25,006)$ were

Sanjay Behari

sbehari27@yahoo.com

1 Department of Neurosurgery, Sanjay Gandhi Postgraduate Institute of Medical Sciences (SGPGIMS), Lucknow 226014, India compared. The study revealed that patients with AS are at a 30-50\% increased risk of incident cardiovascular events [9]. A retrospective cohort study from Quebec, Canada, that included 8616 individuals with AS compared with 50,699 individuals in the general population also revealed that patients with AS are at an increased risk for cardiovascular and cerebrovascular diseases. The risk is much higher in younger patients [2]. Another study from Taiwan, using the database obtained from the complete National Health Insurance (NHI) claim database, showed a 1.9-fold increased risk of developing an ischemic stroke in young patients with AS [10].

The increased propensity of patients with AS to develop a stroke has been attributable to several etiopathological factors. Yet, none of them have an unequivocally proven role in the causation of stroke. The Trial of Org 10172 in Acute Stroke Treatment (TOAST) classification denotes five subtypes of ischemic stroke: (1) large artery atherosclerosis, (2) cardioembolism, (3) small vessel occlusion, (4) stroke of other determined etiology, and (5) stroke of undetermined etiology. All five subtypes of ischemic stroke may occur in patients with AS [11].

Large artery atherosclerosis may be causally related to inflammation that forms an integral component of the pathogenesis of AS. Increased levels of inflammatory markers, such as interleukin-6, C-reactive protein, and tumor necrosis factor alpha, increase atherogenesis and may lead to intimal and media thickening of large- and medium-sized blood vessels [10, 12]. Rheumatological states, such as AS, may cause endothelial injury by immune complex formation and complement activation. The disproportionate frequency of methylenetetrahydrofolate reductase (C677T) gene polymorphism in patients with AS may also be a potential etiopathological factor for the development of stroke in AS. Cardioembolic stroke may be the consequence of cardiomyopathy, valvular heart disease, or aortic insufficiency, which constitute the extra-articular manifestations of AS. Small vessel occlusion may be the result of end artery embolic phenomenon or may be the consequence of inflammation and vasculitis associated with AS [10]. Stroke 
of other determined etiology may result from a much higher prevalence rate of smoking, hypertension, and diabetes mellitus in patients with AS, all of which are factors that increase the risk of stroke $[6,7,10]$. In a more advanced stage of AS, lack of sufficient mobilization and the prolonged recumbency of patients, consequent to pain and stiffness of the ligaments and joints of the spine (that are undergoing progressive ossification); the presence of sacroiliitis, peripheral joint inflammation, and costochondral junction calcification (leading to impaired chest expansion and venous return); and, the prolonged use of steroids to treat joint inflammation may lead to the development of deep vein thrombosis and consequently to venous sinus thrombosis and cortical vein thrombosis. This sets in a cascade of events leading to a higher incidence of stroke in this population of patients. Use of non-steroidal anti-inflammatory drugs to relieve joint pain in AS may cause platelet dysfunction leading to hemorrhagic stroke. Cyclooxygenase-2 selective inhibitors, used to treat patients with inflammatory rheumatic diseases, have been associated with an increased incidence of cardiovascular events in recent clinical trials and observational studies [10].

There are, therefore, numerous previously unresolved issues relating to both the association and the etiopathogenesis of vascular disorders in AS. The addressal of some of these issues makes this nationwide age- and sex-matched longitudinal follow-up study, investigating the risk of development of ischemic stroke in AS, from South Korea by Lee et al., a noteworthy one [13]. The 6-year ischemic stroke incidence rate for 12,988 patients with AS and 64,940 controls was calculated. The ischemic stroke patients were defined by applying the following criteria: (1) International Classification of Diseases (ICD)-10 codes (I63, I64), (2) hospitalization of the patient, and (3) the confirmation of ischemic stroke of the brain by a computed tomographic (CT) or a magnetic resonance imaging (MRI) scan. Subjects in this study were followed from the first occurrence of ischemic stroke to death or the end of follow-up (whichever occurred first). During the follow-up period, there was a statistically significant increase $(P=0.0041)$ in ischemic stroke rate in patients with AS than in the control group [13].

This study has several strengths. The database covered nearly $97 \%$ of the population of the nation, thus obviating the effects of ethnicity within the nation, as well as geographical, climatic, or dietary influences. Recruitment of a large number of cases gives the study an indisputable statistical power [13]. The longitudinal nature of the study, with a long-term follow-up period of 6 years, makes this study even more valuable. The long-term follow-up period and the comprehensive coverage of patients have ensured that none of the patients who suffered from stroke during these 6 years in the entire nation were inadvertently overlooked. Thus, the results of this study bear the closest resemblance to the natural manifestations of the disease as it evolved over time.
A study of this magnitude has its inherent limitations. In fact, in its greatest strength (that of being able to recruit $97 \%$ of the nation's population) also lies its greatest weakness. In this nationwide study, a large number of patients have been recruited based upon their retrospective coding in the national database according to the ICD. It is often difficult for many non-specialist physicians to diagnose the presence of AS accurately, and long delays (from 8 to 11 years) may occur between the symptom onset and the establishment of diagnosis. It would not have been possible for every patient of AS recruited in the national database to be unequivocally diagnosed based on well-defined clinico-radiological and biochemical parameters by experts, strictly adhering to the sacrosanct diagnostic criteria of AS. This could have led to an anomalous representation of the group with AS [2]. Moreover, the traditional vascular risk factors, such as diabetes, hypertension, and dyslipidemia, are disorders with an insidious onset; some asymptomatic vascular risk factors may, therefore, not have been diagnosed [10]. Therefore, the prevalence of vascular risk factors may have been underestimated in both the AS and the non-AS groups.

An interesting limitation inherent in studies of these types where healthy individuals are being compared with diseased individuals is the ascertainment bias [2]. It is more likely that patients with AS, as compared with healthy individuals, will be investigated more thoroughly for the presence of cardiovascular or cerebrovascular diseases. This bias would be inherently more pronounced while dealing with younger individuals. The lack of diagnosis of an underlying cerebrovascular disease in the control population would, therefore, go undetected, thus creating a bias toward a higher prevalence ratio of the disease.

Clinical factors are also instrumental in introducing significant bias. The characteristics of the patients, their propensity to influence the development of stroke, the stage of myelopathy, and the incidence of stroke based upon the disability stratification should have been given. In an advanced stage of disability, it is possible that minor stroke could not be identified because the stroke symptomatologies were overlapped by the significant myelopathic symptoms. It is also possible that a majority of patients in a poor myelopathic grade actually suffered from ischemic stroke not due to the disease per se but due to recumbency-related factors such as the presence of deep vein thrombosis, basal pneumonitis, metabolic syndrome, bed sore, and sepsis. Moreover, apart from the common risk factors such as diabetes mellitus and hypertension, and dyslipidemia, none of the other inflammatory, genetic, clinical, or biochemical factors were evaluated in the study. The site of the major spinal affliction (that is, the presence of a lumbosacral/sacroiliac disease with either mechanical backache or radiculopathy versus the presence of a thoracic, or rarely cervical disease, manifesting as myelopathy) as well 
as the degree of spinal deformity would also have a direct bearing on the propensity of patients with AS to having a stroke. These issues were not addressed in the study. An interesting observation in the study was that, although male patients predominated in the study, female patients with AS had a statistically significant higher incidence rate of stroke when compared to the control population [13]. However, no explanations have been forthcoming in the study regarding this gender predilection for developing stroke in patients with AS.

Surgery in AS is usually indicated for correcting the spinal deformity (that usually results in the spine being in a fixedflexion position), for decompressing a stenosed segment of the spine (that has precipitated neurological deficits), and/or for stabilizing an unstable spine. The surgery may be in the form of osteotomies to correct angular deformities, decompressive laminectomies to relieve compression on the nerve root and thecal sac, or spinal instrumentation and fusion to stabilize the spine [14-16]. Both the anesthesia and surgery may precipitate a stroke. Surgery may also be required for correction of other joint deformities in AS. Yet, the influence of this factor was not addressed in the study. Information regarding the type of surgical intervention performed in this large cohort of patients would have been invaluable not only in instituting preventive measures to obviate the risk of stroke but would also have served as a road map for establishing the indications, timing, and type of interventions required in this relentlessly progressive disease.

There is a saying, "Be prepared for the worst, and you shall never be caught unaware." Based upon this adage, and keeping in view the findings of this study as well as the others $[2,6$, $7,10]$, one should anticipate a higher incidence of stroke in patients suffering from AS. Thus, preventive measures to combat the risk factors that may be responsible for the development of a subsequent stroke in these patients, and adequate and early measures to treat both these factors and the stroke that develops, may well be life- and limb saving in this vulnerable population.

\section{Compliance with ethical standards}

Conflict of interest The authors declare that they have no competing interests.

\section{References}

1. Sidiropoulos PI, Hatemi G, Song IH, Avouac J, Collantes E, Hamuryudan V, Herold M, Kvien TK, Mielants H, Mendoza JM, Olivieri I, Østergaard M, Schachna L, Sieper J, Boumpas DT, Dougados M (2006) Evidence-based recommendations for the management of ankylosing spondylitis: systematic literature search of the 3E Initiative in Rheumatology involving a broad panel of experts and practising rheumatologists. Ann Rheum Dis 65:442452

2. Szabo SM, Levy AR, Rao SR, Kirbach SE, Lacaille D, Cifaldi M, Walter P, Maksymowych MP (2011) Increased risk of cardiovascular and cerebrovascular diseases in individuals with ankylosing spondylitis. Arthritis Rheum 63:3294-3304

3. Mathieu S, Gossec L, Dougados M, Soubrier M (2011) Cardiovascular profile in ankylosing spondylitis: a systematic review and meta-analysis. Arthritis Care Res 63:557-563

4. Ungprasert P, Srivali N, Kittanamongkolchai W (2015) Risk of coronary artery disease in patients with ankylosing spondylitis: a systematic review and meta-analysis. Ann Transl Med 3:51

5. Keller KJ, Hsu JL, Lin SM, Chou CC, Wang LH, Wang J, Bai CH, Chiou HY (2014) Increased risk of stroke among patients with ankylosing spondylitis: a population-based matched-cohort study. Rheumatol Int 34:255-263

6. Behrouz R (2014) The risk of ischemic stroke in major rheumatic disorders. J Neuroimmunol 277:1-5

7. Zhang X, Liu R, Wang J, Zhang Y, Liu Y, Yu Z, Yu S (2016) Risk of premature cerebrovascular disease in patients with ankylosing spondylitis. Acta Reumatol Port 41:322-327

8. Brophy S, Cooksey R, Atkinson M, Zhou SM, Husain MJ, Macey S, Rahman MA, Siebert S (2012) No increased rate of acute myocardial infarction or stroke among patients with ankylosing spondylitis - a retrospective cohort study using routine data. Semin Arthritis Rheum 42:140-145

9. Eriksson JK, Jacobsson L, Bengtsson K, Askling J (2017) Is ankylosing spondylitis a risk factor for cardiovascular disease, and how do these risks compare with those in rheumatoid arthritis? Ann Rheum Dis 76:364-370

10. Lin C-W, Huang Y-P, Chiu Y-H, Ho Y-T, Pan S-L (2014) Increased risk of ischemic stroke in young patients with ankylosing spondylitis: a population-based longitudinal follow-up study. PLoS One 9: e94027

11. Chung JW, Park SH, Kim N, Kim WJ, Park JH, Ko Y, Yang MH, Jang MS, Han MK, Jung C, Kim JH, Oh CW, Bae HJ (2014) Trial of ORG 10172 in Acute Stroke Treatment (TOAST) classification and vascular territory of ischemic stroke lesions diagnosed by diffusion-weighted imaging. J Am Heart Assoc 3(4). https://doi. org/10.1161/JAHA.114.001119

12. Ayas O (2017) Is ankylosing spondylitis a risk factor of recurrent cerebrovascular disease. Phys Med Rehabil Int 4:1115-1116

13. Lee DH, Choi YJ, Han IB, Hong JB, Han KD, Choi JM, Sohn S (2018) Association of ischemic stroke with ankylosing spondylitis: a nationwide longitudinal cohort study. Acta Neurochirur. https:// doi.org/10.1007/s00701-018-3499-7

14. Badve SA, Bhojraj SY, Nene AM, Varma R, Mohite S, Kalkotwar S, Gupta A (2010) Spinal instability in ankylosing spondylitis. Indian J Orthop 44:270-276

15. Behari S, Tungeria A, Jaiswal AK, Jain VK (2010) The "moustache" sign: localized intervertebral disc fibrosis and panligamentous ossification in ankylosing spondylitis with kyphosis. Neurol India 58:764-767

16. Zochling J, van der Heijde D, Burgos-Vargas R, Collantes E, Davis JC Jr, Dijkmans B, Dougados M, Géher P, Inman RD, Khan MA, Kvien TK, Leirisalo-Repo M, Olivieri I, Pavelka K, Sieper J, Stucki G, Sturrock RD, van der Linden S, Wendling D, Böhm H, van Royen BJ, Braun J, Assessment in AS international working group, European League Against Rheumatism (2006) ASAS/EULAR recommendations for the management of ankylosing spondylitis. Ann Rheum Dis 65:442-452 